\title{
Applications of Mitsunobu Reaction in total synthesis of natural products
}

\section{$\underline{\text { Majid M. Heravi }}{ }^{1}$, Nastaran Ghalavand, Manizheh Ghanbarian, Leyla Mohammadkhani}

${ }^{1}$ Department of Chemistry, Faculty of Physics \&Chemistry Alzahra University, Vanak, Tehran, Iran.

\begin{abstract}
The Mitsunobu Reaction allows the conversion of primary and secondary alcohols to esters, phenyl ethers, thioethers and some other compounds. The nucleophile employed should be acidic, since one of the reagents, diethylazodicarboxylate (DEAD) must be protonated during the course of the reaction, preventing from the formation of unwanted side products. In this review, we try to focus on the scope and preparative synthetic applications of Mitsunobu reaction as a key step in the total synthesis of biologically active natural products.
\end{abstract}

Keywords: asymmetric synthesis, inversion of configuration, Mitsunobu reaction, natural products, total synthesis, Chemistry, Synthesis Organometallics, Mitsunobu reactions, Synthesis (chemical) 